Bull. Mater. Sci., Vol. 6, No. 1, February 1984, pp. 39-45.

(C) Printed in India.

\title{
Curie temperatures and $0^{\circ} \mathrm{K}$ magnetic moments of zinc-substituted lithium ferrites
}

\author{
PRAN KISHAN, D R SAGAR, S N CHATTERJEE, L K NAGPAUL \\ and $K$ K LAROIA
}

Solid State Physics Laboratory, Delhi 110007 , India

\begin{abstract}
Because of their several attractive features including relatively high Curie temperatures, substituted lithium ferrites have become important for applications at microwave frequencies. Néel collinear arrangement of spins on $A$ and $B$ sublattices is unable to satisfactorily explain the $0^{\circ} \mathrm{K}$ saturation moments and the observed Curie temperatures of the zinc-substituted lithium ferrites, especially at concentrations of zinc $z>0.3$ in the formula $\mathrm{Li}_{0.5-2 / 2} \mathrm{Zn}_{2} \mathrm{Fe}_{2 \cdot 5-3 / 2} \mathrm{O}_{4}$. Rosencwaig's localized canting model has been extended and used to compute $0^{\circ} \mathrm{K}$ magnetic moments and Curie temperatures of these ferrites with substitution levels up to $z=0.7$. Reasonably good agreement between the calculated and experimental values, both for the $0^{\circ} \mathrm{K}$ magnetic moments and the Curie temperatures, has been obtained using exchange parameters ratios based on the values $J_{a a}=-20^{\circ} \mathrm{K}, J_{b b}=-8^{\circ} \mathrm{K}$ and $J_{a b}=J_{b o}=-29^{\circ} \mathrm{K}$.
\end{abstract}

Keywords. Ferrites; Curie temperature; magnetisation; localised spin canting; exchange parameter.

\section{Introduction}

Though the two sublattice collinear model (Néel 1948) was the first step in understanding the magnetic behaviour of ferrimagnets, it proved inadequate (Gorter 1954; Geller et al 1964) to account for the properties of diamagnetically-diluted systems with spinel and garnet structures. This was particularly so for the $0^{\circ} \mathrm{K}$ magnetization in spinel ferrites in which magnetically active cations were replaced with diamagnetic zinc ions preferentially occupying tetrahedral $A$ sites. Based on collinear model and assuming the cation distribution (Nogues et al 1979) of the formula $\left(\mathrm{Zn}_{z}^{2+} \mathrm{Fe}_{1-z}^{3+}\right)_{A}\left\{\mathrm{Li}_{0}^{1+}{ }_{5-z / 2}^{+} \mathrm{Fe}_{1,5+z / 2}^{3+}\right\}_{B} \mathrm{O}_{4}^{2-}$ the zinc substitutions in lithium ferrite are expected to monotonically increase the net magnetization with increase in the diamagnetic content through the enhancement of the $\mathrm{Fe}^{3+}$ ion concentration of the dominant octahedral $B$ sublattice relative to that of $A$ sublattice. This progressive increase in magnetization is expected to continue with increasing zinc until a point is reached where the negative intersublattice $A-B$ superexchange interaction becomes weaker than one of the two intrasublattice interactions $A-A$ or $B-B$, both of which are themselves negative. The two sublattices then transform into two antiferromagnetic states. Experimentally, however, it is observed that zinc substitutions only at low levels cause enhancement in the magnetization followed by a decrease for amounts larger than $z=0.4$. This discrepancy can be explained by assuming a departure from collinearity leading to some sort of a canted spin arrangement resulting from the zinc induced reduction in the normally dominant antiferromagnetic intersublattice exchange interaction. There now exists considerable experimental evidence (Zhilyakov et al 1977; White et al 1978; Dorman et al 1981; Patton et al 1982) that supports the 
presence of spin canting of octahedral $\mathrm{Fe}^{3+}$ ions in this material. Several canting models have been proposed (Yafet and Kittel 1952; Nowik 1969; Rosencwaig 1970) and in what follows the Rosencwaig localized canting model ( $\mathrm{LCM}$ ) has been adapted to the case of zinc-substituted lithium ferrites.

\section{Extended LCM equations}

LCM is a mathematical representation of the proposal of Geller et al (1964) and takes into account the role of intrasublattice exchange interactions and also the existence of chemical disorder that is present in the ferrimagnets due to random ionic substitutions. According to this model if substitution of diamagnetic ions takes place in the $A$ sublattice, a localised canting with different angles of cant occurs only at the $B$ sublattice and vice versa. The spins of a particular $B$ site $\mathrm{Fe}^{3+}$ ion in the former case see an effective exchange field consisting of the $A$ site intersublattice field which depends on the number of nearest neighbour $A$ site magnetic ions that surround it and a $B$ site intrasublattice contribution which is canted at an average canting angle $\bar{\theta}_{b}$. Rosencwaig's original equations were derived for diamagnetic substitutions in garnets. These equations when adapted for spinels and further extended for zinc substitutions in lithium ferrite, with one significant difference discussed in $\S 3$, can be written as follows:

The local canting angle $\phi_{b}(m, q)$ of a $B$ site $\mathrm{Fe}^{3+}$ ion which is surrounded by $m$ and $q$ nearest neighbour diamagnetic ions in the $A$ and $B$ sublattices respectively is given by

$$
\cos \phi_{b}(m, q)=\frac{\left(1-C \cos \bar{\theta}_{b}\right)}{\left(1+C^{2}-2 C \cos \bar{\theta}_{b}\right)^{1 / 2}},
$$

with

$$
C=\frac{S_{b}}{S_{a}} \cdot \frac{J_{b b}}{J_{a b}}=\frac{\left(n_{b b}-q\right)}{\left(n_{b a}-m\right)} \cdot \delta,
$$

where $S_{a}$ and $S_{b}$ are the vector sums of all the spins of the magnetically active nearest neighbours located on $A$ and $B$ sublattices respectively. $S_{a}=\left(n_{b a}-m\right) S ; S_{b}=\left(n_{b b}-q\right) S$ where for a $\mathrm{Fe}^{3+}$ ion $S=2 \cdot 5 ; n_{i j}$ is the number of nearest neighbour bonds that an ion on sublattice $i$ makes with ions on the $j$ sublattice with $n_{a b}=12, n_{b a}$ and $n_{b b}=6, n_{a a}=4$; $\delta$ is the ratio between intrasublattice $B-B$ exchange parameter $J_{b b}$ and that of intersublattice exchange parameter $J_{a b}\left(=J_{b a}\right)$ and the ratio $J_{a a} / J_{a b}$ is represented by $\gamma$. The average canting angle $\bar{\theta}_{b}$ of the $B$ sublattice is given by

$$
\cos \bar{\theta}_{b}=\sum_{m=0}^{n_{b a}} p_{b a}(m) \frac{\left(n_{b a}-m\right)}{2 \delta \sum_{q=0}^{n_{b b}} p_{b b}(q)\left(n_{b b}-q\right)} .
$$

The summation factor

$$
\sum_{q=0}^{n_{b b}} p_{b b}(q)\left(n_{b b}-q\right)
$$

in the denominator works out to be equal to $k_{b} n_{b b} ; p_{i j}(r)$ is the probability of an $\mathrm{Fe}^{3+}$ ion on sublattice $i$ having $r$ of its $n_{i j}$ neighbours on sublattice $j$ as diamagnetic ions while a fraction $k_{j}$ of the latter is magnetic. For the case under consideration $k_{a}=(1-z), k_{b}=(1.5+z / 2) / 2$ and from binomial distribution function $p_{i j}(r)=\left(\begin{array}{c}n_{i j} \\ r\end{array}\right) k_{j}^{\left(n_{i j}-r\right)}\left(1-k_{j}\right)$. As postulated by Rosencwaig $(1970)$ the individual $\cos \theta_{b}$ values in $(2)$ before multiplication with the probability $p_{b a}(m)$ are always limited 
according to

$$
\frac{\left(n_{b b}-m\right)}{2 \delta k_{b} n_{b b}}=\cos \overline{\theta_{b}} \leqslant 1 .
$$

The $0^{\circ} \mathrm{K}$ magnetic moment $\mu(z, T=0)$ in Böhr magneton is given by

$$
\mu(z, T=0)=10 k_{b} \sum_{m=0}^{n_{b a}} p_{b a}(m) \cos \phi_{b}(m, z)-5 k_{a}
$$

where $\cos \phi_{b}(m, z)$ is obtained from (1) for each $m$ at zinc substitution level $z$ by taking summation over all possible values of $q$ from 0 to $n_{b b}$ and multiplying these with their respective probabilities of occurrence.

The Curie temperatures $T_{\mathrm{c}}(z)$ in ${ }^{\circ} \mathrm{K}$ is given by the expression

$$
\frac{T_{c}(z)}{T_{c}(z=0)}=\frac{[(\bar{S}+1) / \bar{S}]_{z}}{[(\bar{S}+1) / \bar{S}]_{z=0}} \cdot \frac{[-V(z)]}{[-V(z=0)]},
$$

where the net spin $\bar{S}$ per ion per formula unit is given by $\bar{S}=\left(k_{a}+2 k_{b}\right) S / 7$ and the total potential energy $V(z)$ per formula unit is represented by

$$
\begin{aligned}
V(z)= & 2 S^{2} J_{a b}\left[2 k _ { b } \sum _ { m = 0 } ^ { n _ { b a } } p _ { b a } ( m ) \cdot \left\{\left(n_{b a}-m\right) \cos \phi_{b}(m, z)\right.\right. \\
& \left.\left.-k_{b} n_{b b} \delta \cos 2 \phi_{b}(m, z)-\frac{1}{2} k_{a}^{2} n_{a a} \gamma\right\}\right] .
\end{aligned}
$$

The Curie temperature $T_{c}(z=0)$ (Pran Kishan et al 1981) of the unsubstituted lithium ferrite in these equations has been taken as $913^{\circ} \mathrm{K}$.

\section{Results and discussion}

In lithium ferrite though the zinc ions on substitution occupy tetrahedral $A$ sites, there occurs a simultaneous change in diamagnetic $\mathrm{Li}^{1+}$ ion content on $B$ sublattice as well according to the formula given above. The Rosencwaig LC model for doublysubstituted systems (Rosencwaig 1970) should therefore apply. According to the criterion that follows from his arguments the sublattice that has a larger absolute value of the product $l_{i} k_{i} n_{i i} J_{i i}$ where $l_{i}$ is the number of magnetic ions per formula unit in the

\begin{tabular}{|c|c|c|c|c|c|}
\hline$J_{a a}(\mathrm{~K})$ & $J_{b t}\left({ }^{0} \mathbf{K}\right)$ & $J_{a b}(\mathrm{~K})$ & $\delta=\left|\frac{J_{b b}}{J_{a b}}\right|$ & $y=\left|\begin{array}{l}J_{a a} \\
J_{a b}\end{array}\right|$ & Reference \\
\hline$-20 \cdot 0$ & $-8 \cdot 0$ & $-29 \cdot 0$ & 0.28 & 0.69 & Srivastva et al (1979) \\
\hline$-28 \cdot 0$ & $-15 \cdot 0$ & $-34 \cdot 0$ & 0.44 & 0.82 & Dionne (1976) \\
\hline$-19 \cdot 5$ & $-10 \cdot 6$ & $-24 \cdot 0$ & $0 \cdot 44$ & $0-81$ & Rado and Follen (1960) \\
\hline-8.0 & $-8 \cdot 8$ & $-24 \cdot 0$ & $0-27$ & $0 \cdot 33$ & Feldman et al (1979) \\
\hline$\ldots$ & --- & - & 0.33 & 0.36 & White et al (1978) \\
\hline
\end{tabular}
sublattice $i$, has its spins subjected to canting. Using the published values of exchange parameters of lithium ferrite as given in table 1 it can be seen that in the ferrite compositions studied here, the canting if occurs does so on the $B$ sublattice only.

Table 1. Exchange parameters of $\mathrm{Li}_{0.5} \mathrm{Fe}_{2.5} \mathrm{O}_{4}$ 
However, due to random placement of diamagnetic ions in the two sublattices, it is possible that there might be some regions in the crystal where on localised basis the value of $l_{b} k_{b} n_{b b} J_{b b}$ becomes smaller than the corresponding value for the $A$ sublattice. It is, therefore, the latter that should experience canting in that region. To mathematically account for such situations Rosencwaig invoked the concept of localised spin flips that should take place for those $A$ site $\mathrm{Fe}^{3+}$ ions that are either weakly or not at all magnetically bound to the $B$ sublattice while only the spins of $\mathrm{Fe}^{3+}$ ions of the latter experience localised canting. This then necessitates the inclusion of an additional factor $\cos \phi_{a}(t, z)$ in the $A$ sublattice term of equations (3) and (4) with the restriction that $\phi_{a}$ can take only the values 0 or $\pi$. Setting $\cos \bar{\theta}_{a}=1$ the values of $\cos \phi_{a}(t, z)$ can be calculated from an equation similar to equation (1) but adapted for the case of $A$ sublattice.

For an $\mathrm{Fe}^{3+}$ ion of $A$ sublattice there are 12 nearest neighbour $B$ site cations. In the unsubstituted lithium ferrite on the average 9 of these are bonds to $B$ site $\mathrm{Fe}^{3+}$ ions while the number $t$ of the bonds to diamagnetic $\mathrm{Li}^{1+}$ ions is only 3 . But because of random distribution of ions in the $B$ sublattice the actual values of $t$ for individual $A$ site $\mathrm{Fe}^{3+}$ ions may vary from 0 to 12 with the probabilities of their occurrence given by the binomial distribution function. Calculations for $\cos \phi_{a}(t, 0)$ show that while $\phi_{a}(t)$ remains zero for all values of $t$ from 0 to 8 , the probability of $t$ taking a value larger than 8 is practically zero. At higher $z$ levels the concentration of $\mathrm{Fe}^{3+}$ ions on $B$ sublattice increases and the probability of $t$ taking higher values reduces still further. That means no spin flips at all on $A$ sublattice while the spins of $B$ site $\mathrm{Fe}^{3+}$ ions alone experience canting depending on the zinc substitution level of the ferrite composition. Thus for the present series the factor $\cos \phi_{a}(t, z)$ always remains equal to one and need not be included in (3) and (4) and that the Rosencwaig LC equations for singly-substituted systems should apply.

The exchange field seen by a $B$ site $\mathrm{Fe}^{3+}$ ion has contributions arising both from $A$ and $B$ sublattice magnetically active ions. While the $B$ sublattice field experienced by a particular $B$ site $\mathrm{Fe}^{3+}$ ion is canted at an average canting angle $\overline{\theta_{b}}$ which is the same for all the $\mathrm{Fe}^{3+}$ ions of this sublattice, its magnitude depends on the number of other $B$ sublattice nearest neighbour $\mathrm{Fe}^{3+}$ ions that surround it. In singly-substituted garnets with diamagnetic ions replacing $\mathrm{Fe}^{3+}$ ions on the $A$ sublattice dealt in Rosencwaig (1970) this number remains unchanged and therefore the corresponding $D$ sublattice exchange field contribution is the same in magnitude and direction for all the $D$ site $\mathrm{Fe}^{3+}$ ions. For the present series, however, the number of magnetically active $B$ sublattice ions that surround a $B$ site $\mathrm{Fe}^{3+}$ ion changes with $z$ and because of random distribution of ions on the sublattice it may take any value from 0 to $n_{b b}$ with probabilities that can be calculated with the help of the probability equation given earlier. This feature, though unnecessary for the case of a singly substituted garnet mentioned above, has to be incorporated in the canting equations for zinc substitutions in lithium ferrite. For example in ( 2 ) $n_{b b}$, corresponding to Rosencwaig (1970) has been replaced by

$$
\sum_{q=0}^{n_{b b}} p_{b b}(q)\left(n_{b b}-q\right)=k_{b} n_{b b} .
$$

Similar modifications are needed in other equations as well where $n_{b b}$ and $n_{a a}$ have been replaced by $k_{b} n_{b b}$ and $k_{a} n_{a a}$ respectively. It may be mentioned here that modifications on 
the same lines are also necessary in the canting equations proposed in Rosencwaig (1970) for doubly-substituted systems. The $k_{i}$ factors that already appear in these equations account for changes in the number of magnetic ions only on diamagnetic substitution and not in the number of intrasublattice interaction bonds. Without the suggested extension, one can only expect a qualitative agreement between the theory and the experimental results.

In the extended LCM equations given earlier the exchange constant ratios $\delta$ and $\gamma$ are the most important parameters. Several sets of exchange constants as given in table 1 are available in literature for lithium ferrite. Srivastava et al (1979) have shown that a good fit for magnetisation can be obtained with several of these reported sets though that is not so for the susceptibility and that the criterion for the selection of a proper set should be its simultaneous satisfaction of both the data. On this basis these authors determined the exchange parameters for a number of spinels excluding lithium ferrite for which susceptibility data were not available. They, however, chose a set for the latter which was in conformity with the exchange constants of the other similar spinel systems and is thus expected to be the right set for the compound. With this and other sets of exchange constants of table 1 , theoretical values of $5^{\circ} \mathrm{K}$ magnetic moment have been calculated on the basis of LC model for the series of zinc substituted lithium ferrite. These values have been plotted in figure 1 along with experimental results of Gorter (1954). It can be seen that in the substitution range studied here, there is a functional similarity between the experimental data and the calculated values. As expected the agreement is better for the values calculated with exchange parameters reported by Srivastava et al (1979). Considering the limitations of the model, one of which is the assumption that the exchange parameters are taken independent of the extent of diamagnetic substitution, the agreement between the theory and experimental data can be regarded as reasonably good.

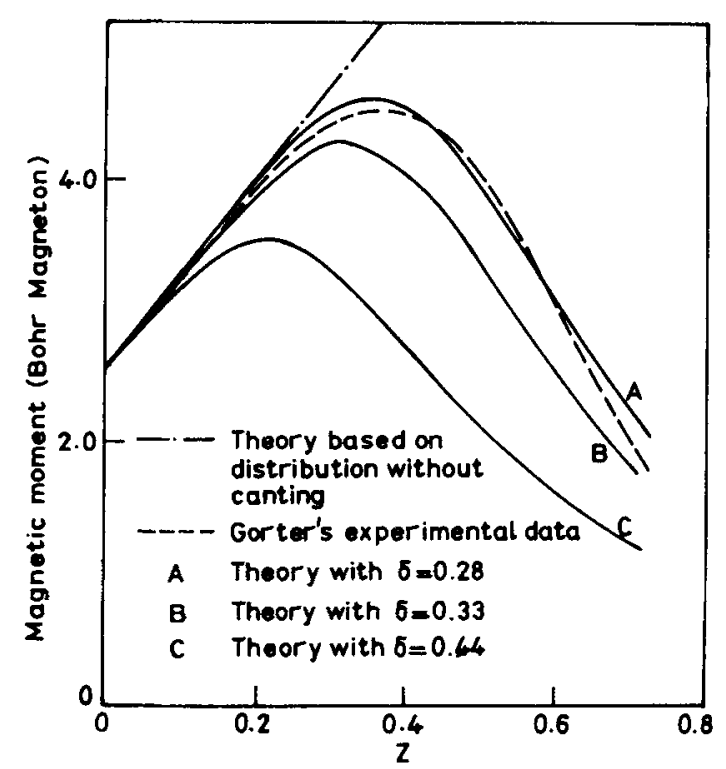

Figure 1. Zero degree $K$ magnetization as a function of $\mathrm{Zn}$ concentration $z$ in $\mathrm{Li}_{0.5-z / 2} \mathrm{Zn}_{z} \mathrm{Fe}_{2 \cdot 5-z / 2} \mathrm{O}_{4}$. 


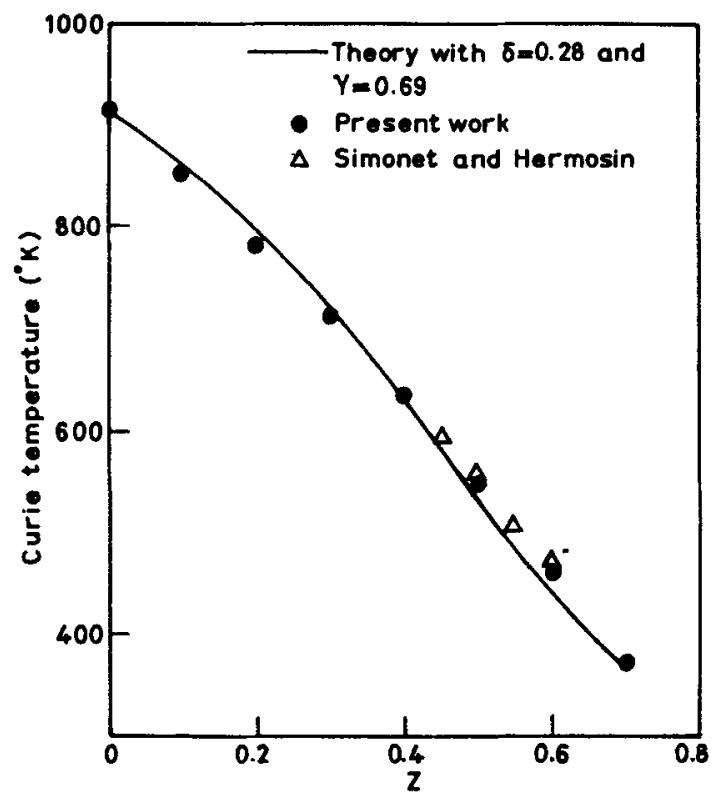

Figure 2. Calculated and experimental Curie temperature for the system with $z=0$ to 0.7 in $\mathrm{Li}_{0.5-2 / 2} \mathrm{Fe}_{2 \cdot 5-2 / 2} \mathrm{O}_{4}$.

The same set of exchange parameters i.e. $\delta=0.28$ and $\gamma=0.69$ was also used in the calculation of Curie temperatures of these compositions. As depicted in figure 2 the agreement is quite satisfactory with the experimental data of Simonet and Hermosin (1978) and that obtained in the present study. The methods of sample preparation and Curie temperature determination used by us have been reported elsewhere (Pran Kishan et al 1981).

\section{Conclusion}

It can be stated that large zinc substitutions in lithium ferrite lead to a noncollinear spin arrangement adequately described by Rosencwaig localized canting model. Furthermore, the set of exchange constants reported by Srivastava et al (1979) for lithium ferrite when used in canting equations predicts reasonably correct values of $0^{\circ} \mathrm{K}$ saturation magnetization and Curie temperatures in zinc-substituted lithium ferrite compositions.

\section{References}

Dionne G F 1976 J. Appl. Phys. 474220

Dorman J L, Merceron T and Nogues M 1981 Applied Mössbauer effect, Proc. Int. Conf., Jaipur

Feldmann P, Simonet W, Desvignes J M and Le Gall H 1979 Joint Intermag 3M Conf. (New York), Abst. 7P10 (Unpublished work)

Geller S, Williams H J, Spinola G P and Sherwood R C 1964 Bell. Sys. Tech. J. 43565

Gorter E W 1954 Phillips. Res. Rep. 9295 
Néel L 1948 Ann. Phys. (Paris) 3137

Nogues M, Dorman J L, Perrin M, Simonet W and Gibert P 1979 IEEE Trans. MAG-15 1729

Nowik I $1968 \mathrm{~J}$. Appl. Phys. 405184

Patton C E, Edmondson C A and Liu Y H 1982 J. Appl. Phys. 532431

Pran Kishan, Chatterjee S N, Nagpaul L K and Laroia K K 1981 Indian J. Pure Appl. Phys. 1983

Rado G T and Follen V J 1960 J. Appl. Phys. 3162

Rosencwaig A 1970 Can. J. Phys. 48 2857, 2868

Simonet W and Hermosin A 1978 IEEE Trans. MAG-14903

Srivastava C M, Srinivasan G and Nanadikar N G 1979 Phys. Rev. B19 499

White G O, Edmondson C A, Goldfarb R B and Patton C E 1978 J. Appl. Phys. 502381

Yafet Y and Kittel C 1952 Phys. Rev. 87290

Zhilyakov S M, Ivolga V V, Mel' tsev V I and Naiden E P 1977 Sov. Phys. Sol. State 191817 